\title{
Design of Fuzzy Enhanced Hierarchical Motion Stabilizing Controller of Unmanned Ground Vehicle in Three DimensionalSpace
}

\author{
Yue Ma* \\ School of Mechanical Engineering, Beijing Institute of Technology, 5 South Zhongguancun Street, Beijing, CHINA, \\ 100081 \\ Changle Xiang \\ Beijing Institute of Technology, 5 South Zhongguancun Street, Beijing,CHINA, 100081
}

Quanmin Zhu

Bristol Institute of Technology, University of the West of England (UWE), Frenchay Campus, Coldharbour Lane, Bristol BS16 1QY, UK

Qingdong Yan

Beijing Institute of Technology, 5 South Zhongguancun Street, Beijing,CHINA, 100081

\author{
Alan Winfield \\ Bristol Institute of Technology, University of the West of England (UWE), Frenchay Campus, Coldharbour Lane, Bristol \\ BS16 1QY, UK \\ Received 13 September 2011 \\ Accepted 25 November 2011
}

\begin{abstract}
In this paper, stabilizing control of tracked unmanned ground vehicle in 3-D space was presented. Firstly, models of major modules of tracked UGV were established. Next, to reveal the mechanism of disturbances applied on the UGV, two kinds of representative disturbances (slope and general disturbances in yaw motion) were discussed in depth. Consequently, an attempting PID method was employed to compensate the impacts of disturbances andsimulation results proved the validity for disturbance incited by slope force, but revealed the lack for general disturbance on yaw motion. Finally, a hierarchical fuzzy controller combined with PID controller was proposed. In lower level, there were two PID controllers to compensate the disturbance of slope force, and on top level, the fuzzy logic controller was employed to correct the yaw motion error based on the differences between the model and the real UGV, which was able to guide the UGV maintain on the stable state. Simulation results demonstrated the excellent effectiveness of the newly designed controller.
\end{abstract}

Keywords: Unmanned Ground Vehicle, Hierarchical Control, Fuzzy Logic Enhanced, Stabilizing, Ground Disturbances, 3-D Space

\section{Introduction}

The unmanned vehicle become more and more important in present world, which have acted as explorers, rescuers, detectors, servants[1]. As
trackedUGV has plenty of prominent advantages in the practical area, lots of subject of research focus on it. As a UGV mainly works in somewhat self-organized manner, consequently, knowing where it is, where the destination is, how it can get there and keeping stability when it is running are important for a UGV. 
Furthermore, when a UGV works outdoors and has to be subject to space constraints in 6-DOFs, in which environment, lots of disturbances make the objects above difficult to realize.Disturbances could be summarized as follow:

(a) Varied adherence coefficient of varied type of ground,

(b) Varied resisting force on magnitude and direction coming from varied terrain,

(c) Random disturbance under uncertainty environment.

Under the effects of disturbances, $\mathrm{UGV}$ is prone to deviate from predefined routine, which would lead UGV into uncertainty and dangerous situation in all probability.That is, stability of motion trajectory of UGV is damaged.

With regard to theoretical level, general motion stability theory [2] elaborate on the rules which a dynamic system should respect to keep itself stabilizing. As it is a very important property for most of dynamic systems, theories of stability of motion were applied broadly, for instance, in general plants or mechanical systems to keep the system stabilize to equilibrium working point[3-5].

In the domain of ground vehicle, automotive industry paid more attention to the handling stability when car is running in curve path with high speed[6-8]. Based on the analysis of stability, driving assistant system, such as electronic stability program (ESP) was developed rapidly and became the standard equipment of car as a matter of fact $[9,10]$. Without ESP, when car is running with left wheel on icy road and right wheel on blacktop, side slip would drive the car overturn. Equipped with ESP, under the same condition above, ESP would reallocate driving force to left and right wheel to keep car running stable.

If we look through the discussions presented above on the theory of stability and its applications, the concept of stability has been defined as the convergence to the zero of error between the motion states and that of expected parameters of plants in pure mathematical definition; and under the same definition, the motion stability of UGV can be summarised as the error between the motion states and pre-defined motion states have to be converged to zero with time goes on.

In this paper, models of major modules of UGV were established.Next, to reveal the mechanism of disturbances applied on UGV, two kinds of representative disturbances (slope and general disturbances in yaw motion) will be discussed in depth, which will be the fundamental objects to be resolved. Consequently,based on the condensed review on fuzzy logic theory, an attempting PID method will be employed to compensate the impacts of disturbances; finally, a hierarchical fuzzy-PID controller will be proposed to improve the motion stability.

\section{Modelling of UGV}

Unexceptionally, modelling is the first key procedure in research of UGV, which is the foundation for the subsequent research, i.e. PID and fuzzy logic controller design.

\subsection{Scheme ofUGV}

Generally speaking, a UGV is a class of complex systems consisting of lots of mechanical, electric and electronic parts. Fig.1present a scheme of UGV designed by Beijing Institute of Technology, which is involved the following modules according the function: (1) energy source module, (2) control module, (3) power and driving module, (4) main body and (5) auxiliary module.

In this paper, models of driving module and main body are built up, and others are neglected because they contribute tiny affection on the dynamic behaviour of UGV in short period.

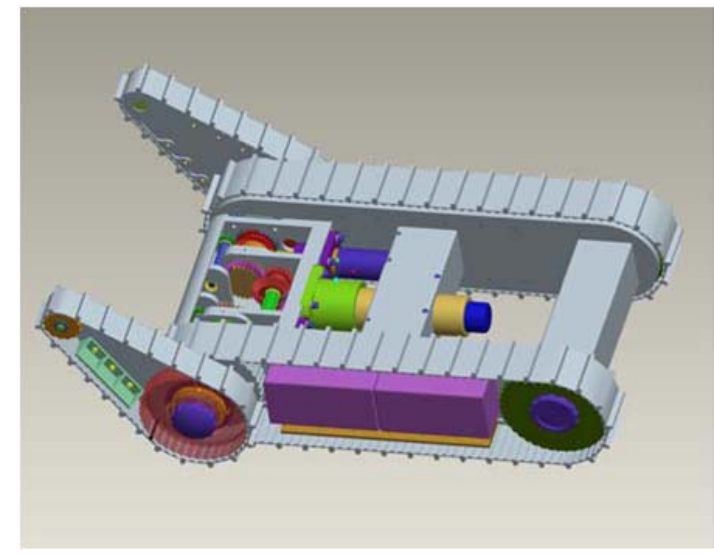

Fig.1. Prototype of Miniature UGV

\subsection{Modelling of Main Body}

Compared with most of research proposed by staff in robotics and vehicle industry, which are carried out in 2-D space, body model of UGV is described in 3-D space in this paper. And in this condition, motions in 
six-DOFs should be considered. According to NewtonEuler formulation, the dynamic equations of UGV designed in my lab should be:

$$
\delta m \frac{d \mathbf{V}}{d t}+\delta m \tilde{\boldsymbol{\omega}} \mathbf{V}=\left[\begin{array}{c}
F_{t 1}+F_{t 2}-F_{f}-m g \sin \theta \\
F_{y}+m g \cos \theta \sin \phi \\
N-\cos \theta \cos \phi m g
\end{array}\right]
$$

where:

$$
\begin{gathered}
\mathbf{V}=\left[\begin{array}{l}
v_{x} \\
v_{y} \\
v_{z}
\end{array}\right] \\
\tilde{\boldsymbol{\omega}}=\left[\begin{array}{ccc}
0 & -\omega_{z} & \omega_{y} \\
\omega_{z} & 0 & -\omega_{x} \\
-\omega_{y} & \omega_{x} & 0
\end{array}\right] \\
{\left[\begin{array}{ccc}
I_{x} & 0 & 0 \\
0 & I_{y} & 0 \\
0 & 0 & I_{z}
\end{array}\right] \frac{d \boldsymbol{\omega}}{d t}+\tilde{\boldsymbol{\omega}}\left[\begin{array}{ccc}
I_{x} & 0 & 0 \\
0 & I_{y} & 0 \\
0 & 0 & I_{z}
\end{array}\right] \boldsymbol{\omega}} \\
{\left[\begin{array}{ccc}
0 & \\
0 & \\
\left(F_{t 2}-F_{t 1}\right) \frac{B}{2}-\operatorname{sign}\left(M_{r}\right) M_{r}
\end{array}\right]} \\
\boldsymbol{\omega}=\left[\begin{array}{l}
\omega_{x} \\
\omega_{y} \\
\omega_{z}
\end{array}\right]
\end{gathered}
$$

in (1), $F_{f}$ stands for deformed resistance of ground, $m$ stands for mass of mobile robot and $\delta$ is named as gain coefficient of mass, which converts the rotational inertia equally to translational mass, dimensionless. For a general mechanical system with some rotational components, in general, $\delta=1.1 \sim 1.2$ [11]. Subsequently, $\mathrm{F}_{y}$ is the forces in $\mathrm{y}$ axis direction, in fact, it is a centripetal force.

And in (4) and (5), $\omega_{z}$ stands for angular velocity of $\mathrm{UGV}$ relative to axis $z ; \alpha_{z}$ stands for angular acceleration of UGV relative to axis $z ; I_{z}$ is rotational inertia of $\mathrm{UGV}$ according to axis $z, M_{r}$ is resistant torque applied on $\mathrm{UGV}$; and finally, $B$ is the distance between the two centres of tracks. Finally, $\theta$ and $\phi$ stand for the pitch and roll angle of the UGV body fixed frame referred tothe global frame respectively.

Furthermore, the deformed resistance of ground, $F_{f}$ is the function of normal pressure of ground

$$
F_{f}=f N_{z}
$$

Where $f$ denotes the coefficient of deformed resistance. $N_{z}$ is the dot product of vector of gravity and the normal vector of contact area

$$
\begin{aligned}
N_{z} & =-m g \mathbf{K} \cdot \mathbf{k} \\
& =m g \cos \theta \cos \phi
\end{aligned}
$$

As to the another important parameter, resistant torque, $M_{r}$, is the function of $m g, L, B$ and the attitude angles of UGV, which will be discussed in the following section.

\section{Disturbances on UGV}

When a UGV is running outdoors, many kinds of disturbances coming from slope and rugged surface will affect its motion status, and furthermore, on exceeding some limits, motion stability of UGV will be challenged immensely. Further research reveals that there are three types of disturbances according to the mechanism of disturbances: (1) centrifugal force, (2) slope force and (3) general disturbances on yaw motion.

As the affection of centrifugal force was discussed in other papers andyielded minor effect on the miniature UGV. Therefore, only effects of slope force and general yaw motion are discussed here.

Slope force is a reacting force, which is to counteract the effect of the gravity driving UGV down slope. As in this research, the UGV mainly works in 3-D space; therefore, the slope force cannot be ignored.

General disturbance on yaw motion of UGV is the abstract disturbance of several kinds of disturbances. If disturbances are applied on the two tracks of the UGV equally to, then, the yaw angle will not be affected and the UGV can keep stable in the direction of motion. Unfortunately, UGV seems never so lucky to get balanced loads on two tracks. Difference of the coherent status of the two tracks, difference of the shape of terrain under the two tracks, as well as random lateral resistant force, can yield additional disturbances on the yaw motion of the UGV, which have the same feature that all can be denoted as the disturbances on the angular acceleration.

Before going into detailed discussion, the following hypotheses are necessary:

The surface of ground is large enough to assure the contacting surface of tracks are local planar, hence, in spite of the variation of curvature of ground, the local 
area where UGV locates is considered as local plane;Suppose that lateral motion of UGV is in steady state, without any acceleration.

Effects of slope force are not only in the lateral direction of UGV but also in the longitudinal direction, and the magnitude is time-variable:

$$
F_{t}=m g \cos \theta \sin \phi
$$

Accordingly, incited offset can be yielded:

$$
O F=\frac{m g \cos \theta \sin \phi L}{2 \mu_{t} N_{z}}
$$

and steady lateral velocity

$$
\begin{aligned}
v_{y} & =\omega_{z} \times O F \\
& =\frac{m g \omega_{z} \cos \theta \sin \phi L}{2 \mu_{t} N_{z}}
\end{aligned}
$$

As the $F_{t}$ also incites the rearrangement of lateral resistant shearing stress, then:

$$
M_{r}=\frac{\mu_{t} m g L \cos \theta \cos \phi}{4}+\frac{m g \cos \theta \sin \phi \tan \phi L}{4 \mu_{t}}
$$

From(10), if UGV doesn't rotate, then, no $v_{y}$ appears, which means that the slope force doesn't affect the stability of straight-line motion. Consequently, the behaviours of UGV in two turning processes under the disturbance of slope force are investigated in MATLAB/Simulink, one is started from the initial condition of $\phi=\phi_{s}=\pi / 60\left(3^{\circ}\right), \theta=\psi=0$, and the other is started from the initial condition of $\phi=\phi_{s}=\pi / 30\left(6^{\circ}\right), \theta=\psi=0$. Fig.2 illustrates the results of $\phi_{s}=6^{\circ}$ ( $\left.n_{m 1}=3000 \mathrm{r} / \mathrm{min}, n_{m 2}=1000 / r \min \right)$ and Fig.3 illustrates the results of $\phi_{s}=3^{\circ}$ $\left(n_{m 1}=3000 \mathrm{r} / \mathrm{min}, n_{m 2}=1000 / \mathrm{r} \min \right)$.

From Fig.2and Fig.3, significant deviation along the direction of slope force appeared after fifty seconds running. The greater the slope angle is, the more considerable the deviation is. On $\phi_{s}=3^{\circ}$ slope, the deviation reaches the $0.2510 \mathrm{~m}$; while, when slope angle is $6^{\circ}$, the maximum deviation between expected curve and real curve is $0.5836 \mathrm{~m}$. Without a doubt, for UGV, results presented above are definitely unacceptable.

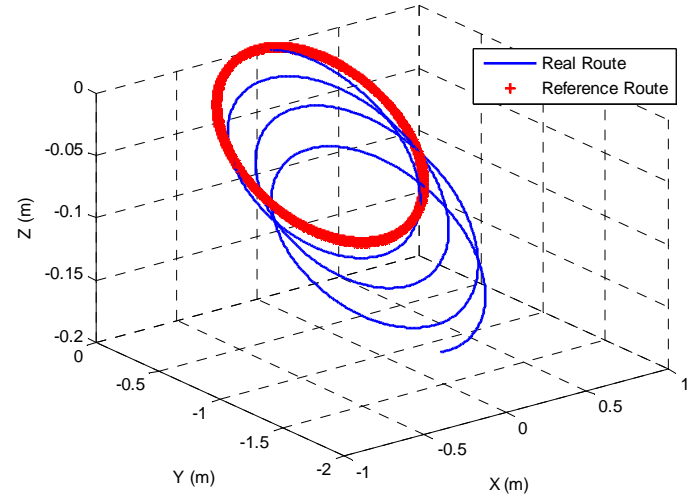

Fig.2. Space curve of UGV on slope $\phi_{s}=6^{\circ}$

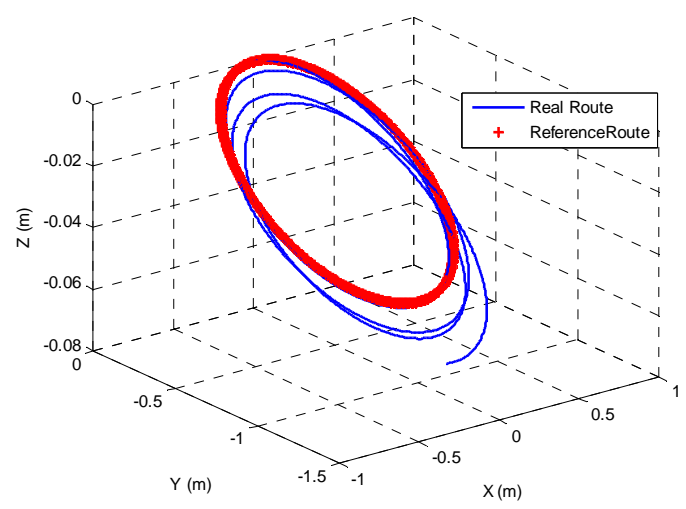

Fig.3. Space curve of UGV on slope $\phi_{s}=3^{\circ}$

Disturbances mentioned above are connatural for UGV in 3-D space, while some random disturbances in motion also make UGV deviate from stable state. For example, when a $U G V$ is running on rough terrain, if track in one side is running over a flat surface while track on the other side has to overcome a small obstacle with the same speed, then in fact, the UGV undertakes a small turning in this procedure in yaw direction; another probable situation is that $\mathrm{UGV}$ is running on a planar surface but there are two diverse contacting conditions under the two tracks, for instance, one is slippery and the other is solid, in which situation the UGV leads to turn to the direction of slippery side, then, unexpected yaw motion occurs. Definitely, there are many other disturbances which will make UGV rotate in yaw direction with vastly different disturbing mechanism. It is a great challenge to establish mechanics model for every disturbance. On analysing the behaviour of these disturbances, the most common feature is the result of disturbances can be reflected by the rate of variation of angular velocity in axis $z$ (i.e. 
angular acceleration in axis $z$ ). Therefore, in this research, all of disturbances in this conditions are converted into the noise angular acceleration in axis $z$. To clarify the effects of general yaw motion disturbance, simulation was carried out, in which UGV was supposed to follow a straight line coincided with axis $x$ in inertia coordinates. The disturbance applied on UGV is illustrated in Fig. 4, and the effects on route of UGV are shown in Fig. 5.

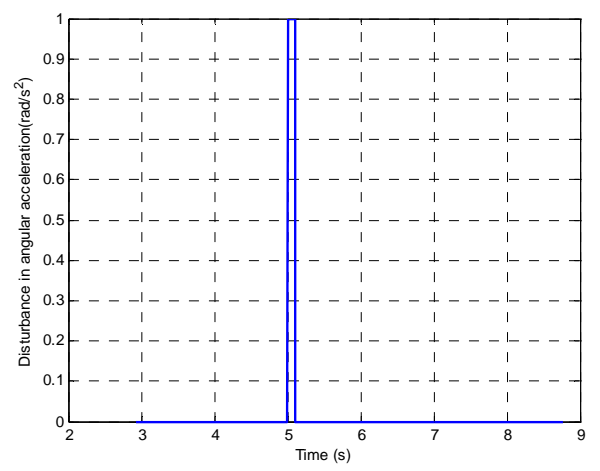

Fig.4. Disturbance in angular acceleration

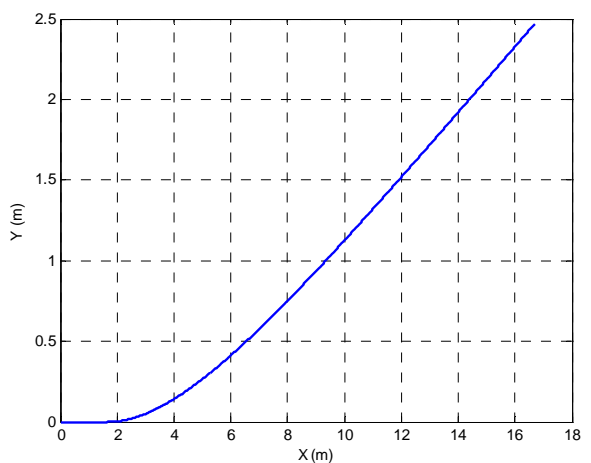

Fig.5. Disturbed route of UGV

\section{Discussions:}

From figures above, the effects of slope force are significant: the real route of UGV is obviously deviated from the reference route; and when $\mathrm{UGV}$ is subjected to the general yaw motion disturbance, real route will deviate from predefined route rapidly. From Fig. 5, only after nine seconds running, the deviation between predefined routine (axis $\mathrm{x}$ ) and real routine increased to $2.4 \mathrm{~m}$, which affected the stabilizing performance and of UGV enormously. Therefore, controller design to improve the ability of keeping stability is necessary and indispensable.

\section{Design of PID Based Motion Stabilizing Controller of UGV}

Based on the discussion above, to keep the motion stability of UGV, all of disturbances should be compensated by a controller appropriately, which is named as stabilizing controller. Starting from convenience and simplicity, the first choice for attempt is employing a PID controller, which is the most universal but powerful tool for control engineering.

There are two necessary functions or modules which should be realized by the designed stabilizing controller: Motion control module (path following and/or attitude control): Firstly, stabilizing controller accepts the instructions from planning or navigation module of UGV, which are the descriptions on status ( path and/or attitude ) UGV should be in; and in general; the path curve is differentiable. Secondly, on receiving data of motion sensors, stabilizing controller manages to map the path and/or attitude information into expected rotational speed of motors for driving; and finally, transfer the motion instructions to the controller of motors to complete a control cycle.

Compensation module based on estimation of disturbances: Obviously, effects of disturbances can be represented in the variation of parameters of motion (such as curvature of path), and can be adapted by the motion control module. But, as the control system and driving system must take a short period of time to response the disturbances passively, therefore, stability performance will be depraved. Thus, active compensations based on the estimation of disturbances are preferred to improve the efficiency of stabilizing controller.

Integration of motion control module and compensation module: Based on the modules mentioned above, an integration module should be presented to compose the final control output advisably.

As the most extensive control algorithm, PID may be the first choice for all kinds of control issues, as well as in mobile robot [12-16]. In this research, a digital PI controller is employed as a motion control module, and a digital $\mathrm{P}$ controller is for the compensation module, which is illustrated in Fig. 6. 


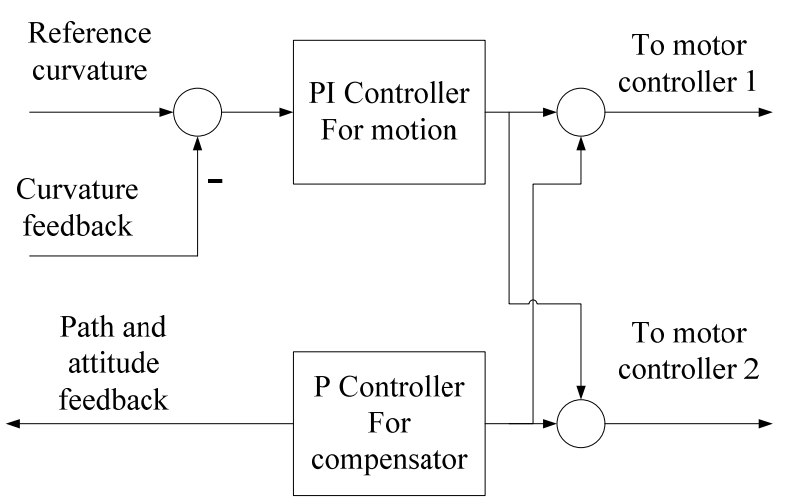

Fig.6. Schema of PID controller

In this project, there is no direct information of position for motion controller, and the shape of path will be the key parameter. In the motion control module, the curvature of the path is selected to be the path reference input, which is most convenient for representing some specific curves utilized in path planning. For instance, the curvature of a straight line is 0 and that of a circle with radius of $r$ is $1 / r$, and other complex curves can be presented by the combination of straight line and parts of circle.

Correspondingly, the discrete PID controller is a direct conversion from continuous PID controller, which can be expressed as follows

$$
G(z)=K_{p}\left(1+\frac{T z}{T_{i}(z-1)}+\frac{T_{d}(z-1)}{T z}\right)
$$

where $T$ is the sampling time.

In compensation module, a $\mathrm{P}$ controller is designed as

$$
\text { Output }=K_{\phi} \omega_{z} \phi \cos 2 \phi
$$

where

$K_{\phi}$ stands for proportional gain of inclined slope $\phi$ is the inclined angle of slope

The implemented controller is shown in Fig. 6.

\section{Simulation Results undertheControl of PIDController}

On designed the two modules, simulations are carried out. Firstly, situation under disturbance incited by slope force is executed. Fig. 7 and Fig. 8show the control results on slope of $3^{\circ}$, and Fig. 9 and Fig. 10 present the control results on slope of $6^{\circ}$.

Secondly, simulations under the general disturbance of yaw motion on plane surface are put forward with the same disturbing noise illustrated in Fig. 4 and the results are shown from Fig. 11andFig. 12.

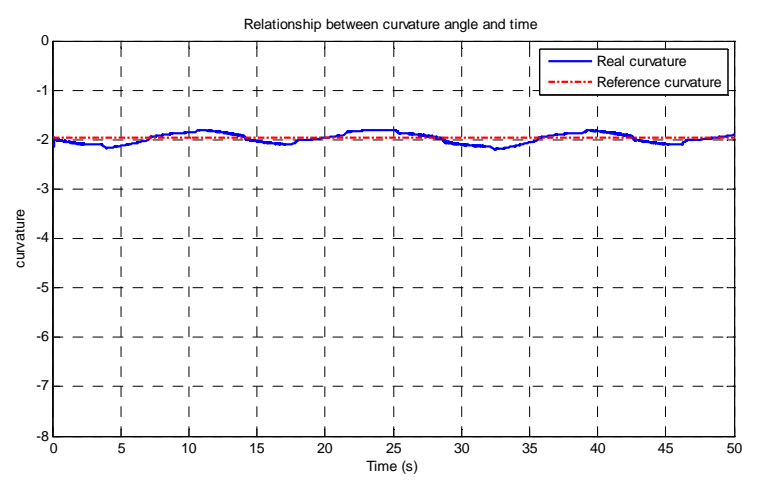

Fig.7. Comparison of real curvature and reference curvature on 3 degree slope

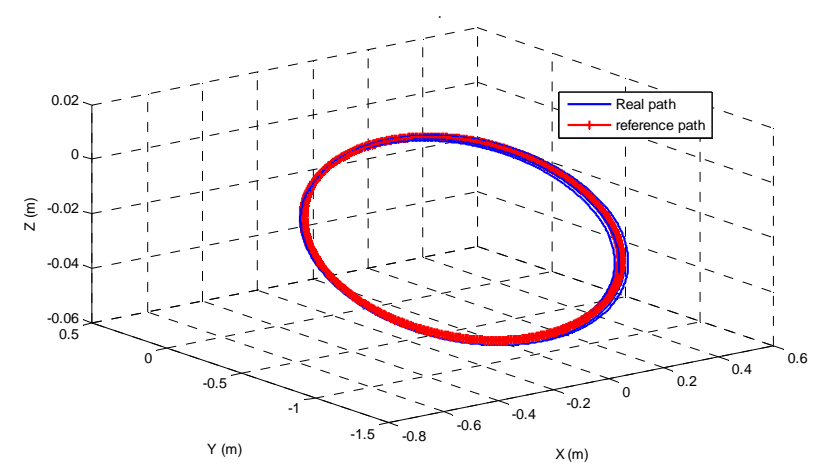

Fig.8. Comparison of real path and reference path on 3degree slope

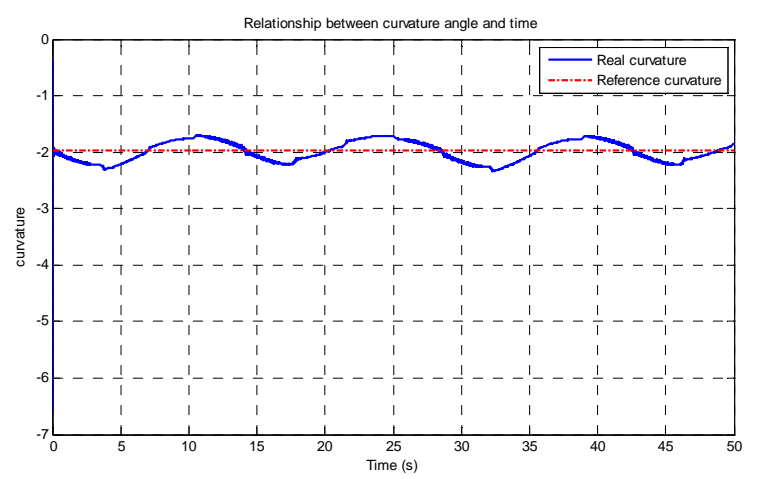

Fig.9. Comparison of real curvature and reference curvature on 6 degree slope 


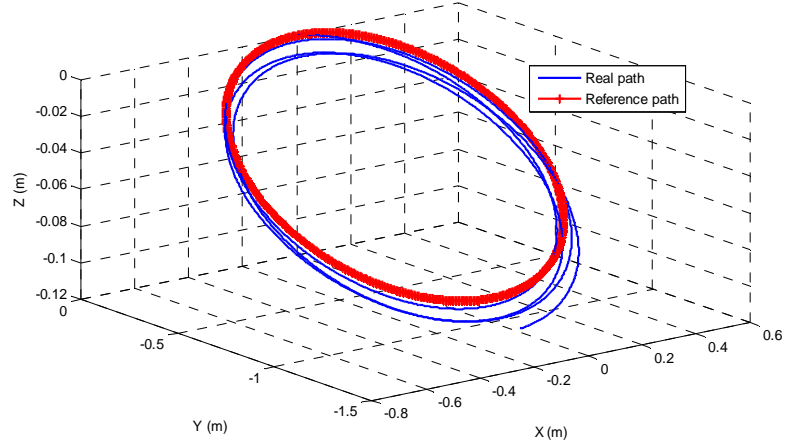

Fig.10. Comparison of real path and reference path on 6 degree slope

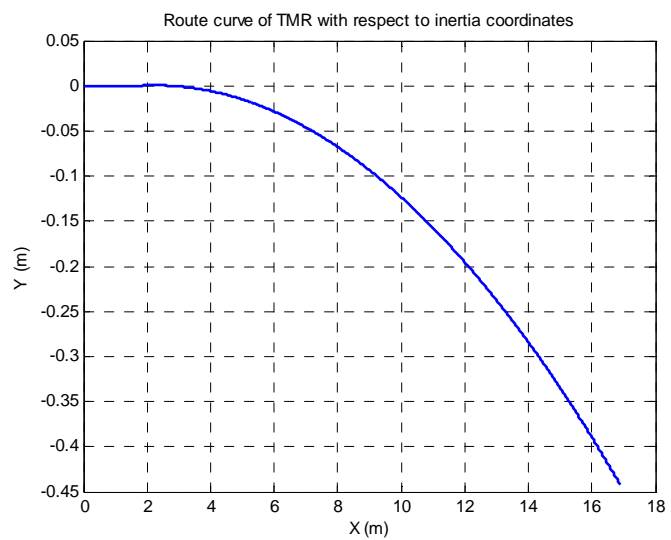

Fig.11. Comparison of real path and reference path on straight-line running

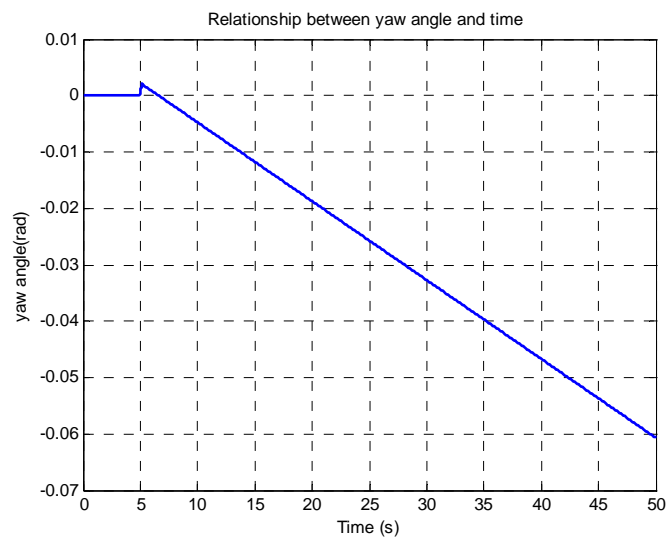

Fig.12. Relationship of yaw angle $\psi$ and time

Discussions:

For a PID controller (PI plus P control in fact), the control effects on motion stabilizing are significant (compared with Fig.2 and Fig. 10, or Fig. 3 and Fig. 8).
When slope angle is $3^{\circ}$, the maximum deviation between expected curve and real curve is $0.0211 \mathrm{~m}$ under the PI control, while the that of situation without control is $0.2510 \mathrm{~m}$; when slope angle is $6^{\circ}$, the maximum deviation between expected curve and real curve is $0.1215 \mathrm{~m}$ under the PI control, while the that of situation without control is $0.5836 \mathrm{~m}$.

The control effects under the general disturbance of yaw motion are interesting. On one hand, the controller did correct or compensate the disturbance on the angular velocity (the deviation after 50 seconds is -0.44 $\mathrm{m}$; while that without control is $2.44 \mathrm{~m}$ and become infinite as time is elapsing); on the other hand, it overdoes in some degree and the new steady state of UGV deviates from the origin of yaw motion obviously (Fig.11).

Above all, the motion stabilizing controller based on PID approach can achieve fairish effects, but it seems that some intelligence is necessary to compensate the general disturbance in yaw motion.

\section{Design of Fuzzy EnhancedMotion Stabilizing Controller of UGV}

According the discussions above, digital PID controller had done its best to reduce the negative effects of disturbances, but, obviously, more intelligence was necessary to achieve our goal, which seems to be lack of brightness like human being. Based on the condensed review on the theory of fuzzy logic, in this paper, a fuzzy logic controller will be proposed which is not a substitution of the PID controller but an integrated hierarchical controller combined with the controller designed in the previous section, because the fuzzy logic controller is to enhance the intelligence while the effects on basic compensating jobs by PID controller are passable. The schema of hierarchical controller is shown in Fig. 13.

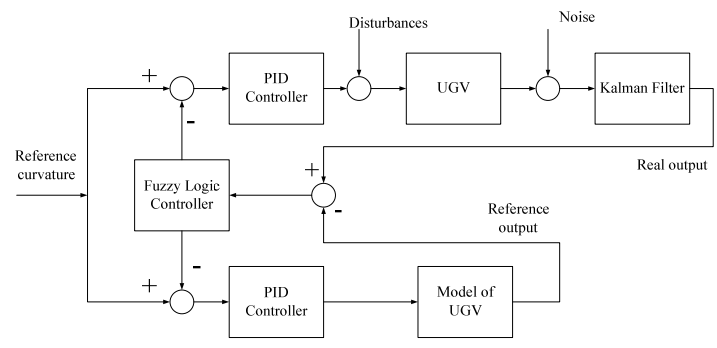

Fig.13. Schema of hierarchical control 
In the hierarchical schema, the path instructions sending to the stabilizing controller are denoted as curvature; both the PID controller of UGV and that of model receive the curvature instructions. If the real UGV get disturbed, then the differences between real UGV and model of UGV will be feedback to the fuzzy controller; consequently, fuzzy controller will compute and generate the revised curvature, and finally transmit the revisions to the PID controller of real UGV.

The fuzzy logic controller has two input parameters, one is the deviation of yaw angle (DYA), and the other is the variation rate of yaw angle (DDYA). The linguist Table 1, and the rules surface is illustrated in Fig. 14. variables of DYA are negative big (NB), negative small (NS), zero (ZO), positive big (PB) and positive small (PS); the linguist variables of DDYA are the same with DYA.

The linguist variables of output are negative big (NB), negative medium (NM), negative small (NS), zero (ZO), positive big (PB), positive medium (PM) and positive small (PS).

The mamdani method is selected as the inference approach of fuzzy logic, table of inference rules is shown in

Table 1Inference rules

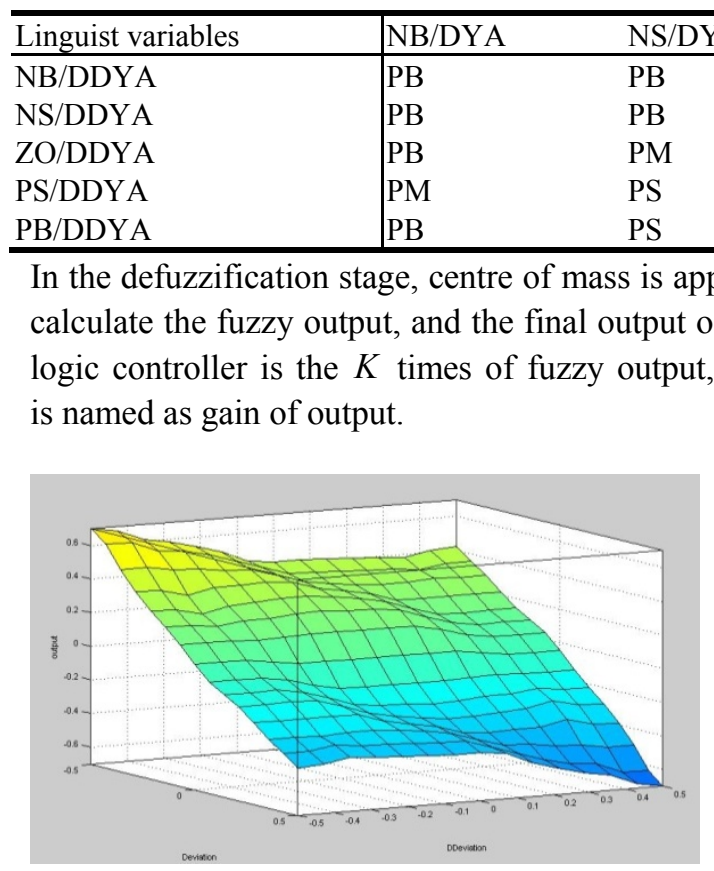

Fig. 14 Surface of inference rules

\section{Design of KalmanFilter}

From the section VI, the fuzzy controller needs two parameters for real-time control, deviation of the yaw angle and the deviation rate of the yaw angle. Generally speaking, a gyro does can supply the two parameters, but there are definitely lots of stochastic noises in the feedback signals of the gyro, therefore, the use of filter is necessary. Kalman filter is prominent in the area of optimum system estimation with stochastic noise[17]. In this paper, a discrete Kalman filter was employed:

\begin{tabular}{ll} 
PS/DYA & PB/DYA \\
NS & NB \\
NS & NM \\
NM & NB \\
NB & NB \\
NB & NB \\
\hline
\end{tabular}

$$
\begin{aligned}
& {\left[\begin{array}{l}
\psi(k+1) \\
\omega(k+1)
\end{array}\right]=\left[\begin{array}{ll}
1 & T \\
0 & 1
\end{array}\right]\left[\begin{array}{l}
\psi(k) \\
\omega(k)
\end{array}\right]+\left[\begin{array}{l}
T^{2} / 2 \\
T
\end{array}\right] v(k)} \\
& y(k)=\left[\begin{array}{ll}
1 & 0 \\
0 & 1
\end{array}\right]\left[\begin{array}{l}
\psi(k) \\
\omega(k)
\end{array}\right]+\left[\begin{array}{l}
0 \\
1
\end{array}\right] w(k)
\end{aligned}
$$

in which: $\psi$ and $\omega$ are yaw angle and angular velocity related to axis $\mathrm{z}$ respectively, and $\mathrm{T}$ stands for the sampling period. $v(k)$ stands for the angular acceleration disturbance in yaw motion on time $\mathrm{k}$ with the covariance $R_{v}$. Finally, $w(k)$ stands for the measurement noise on time $\mathrm{k}$ with the covariance $R_{w}$. Therefore, the gain of the best observer should be:

$$
L(k)=A P(k) C^{T}\left(R_{w}+C P(k) C^{T}\right)^{-1}
$$

where

$$
\begin{gathered}
\begin{array}{c}
P(k+1)= \\
\quad(A-L C) P(k)(A-L C)^{T} \\
\quad+F R_{w} F^{T}+L R_{w} L^{T}
\end{array} \\
P(0)=E\left\{x(0) x^{T}(0)\right\} \\
F=\left[\begin{array}{l}
T^{2} / 2 \\
T
\end{array}\right]
\end{gathered}
$$




\section{Simulation Results undertheControl of Fuzzy Controller}

As the major function of fuzzy logic controller is to compensate the general disturbance of yaw motion, therefore, the performance anti-yaw disturbance of controller is the kernel content of this section.

According to the gain of output $K$, four simulations are carried out. Fig. 15 to Fig. 20 illustrate the results when $K$ equals to 10,15 and 20 respectively. Compared with the results of PID controller, the effects of antidisturbance are improved greatly. For $K=10$, the error between the yaw angle of model and that of real UGV is relative large than others. It seems that the effects of $K=15$ and $K=20$ are both acceptable, but referred to the error of the yaw angle, as the error of yaw angle is convergent to zero when $K$ equals to 15 , therefore, the output gain of $K=15$ is more preferred, in which condition, the maximum error between expected curve and real curve is $-0.01 \mathrm{~m}$, and the trends is approaching to a steady state after 50 seconds' running.

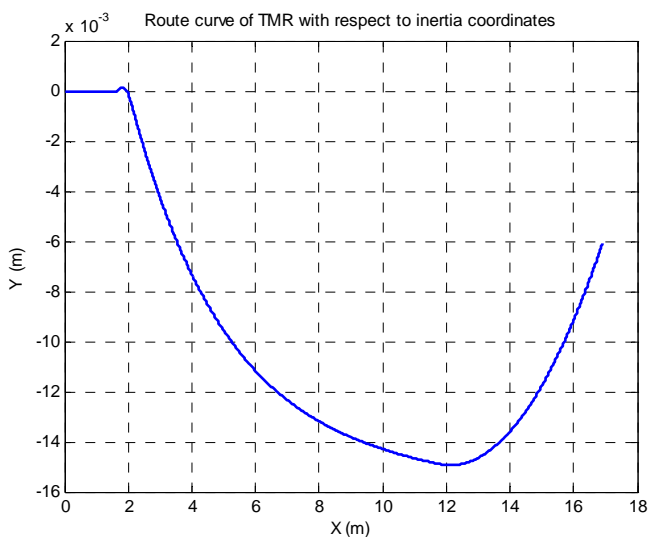

Fig.15. Results of path for $\mathrm{K}=10$

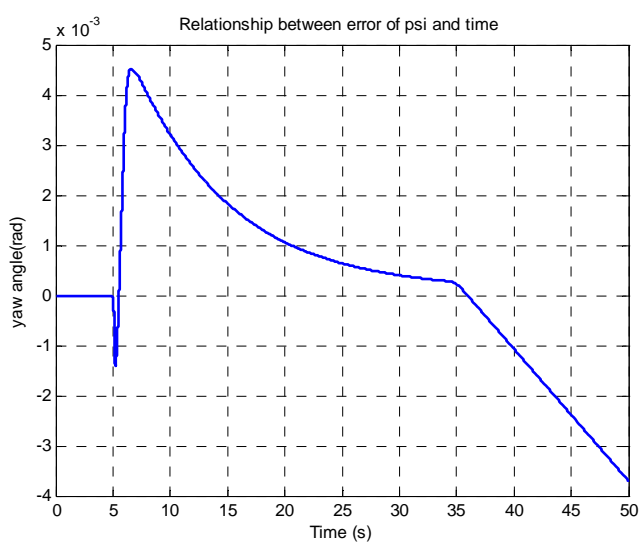

Fig.16. Results of error of $\psi$ for $\mathrm{K}=10$

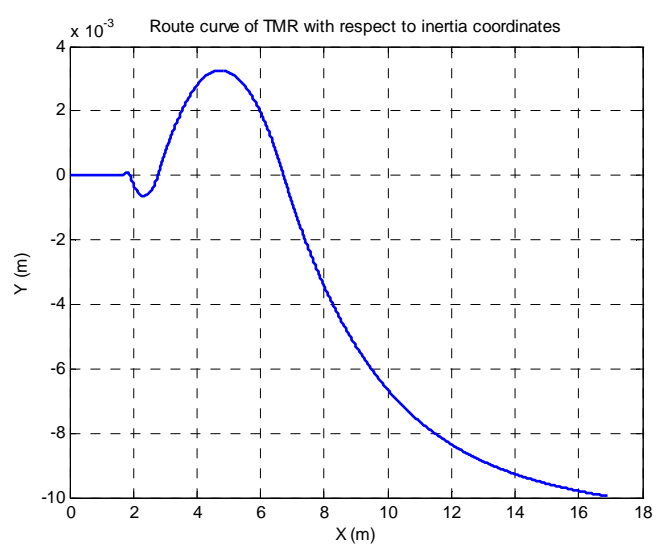

Fig.17. Results of path for $K=15$

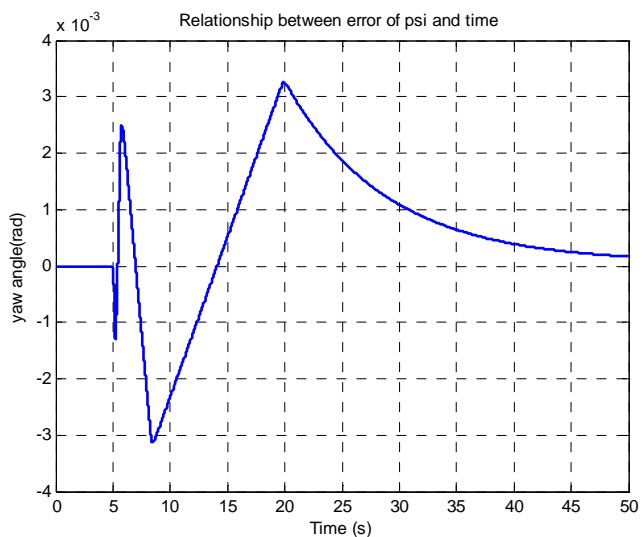

Fig.18.Results of error of $\psi$ for $K=15$ 


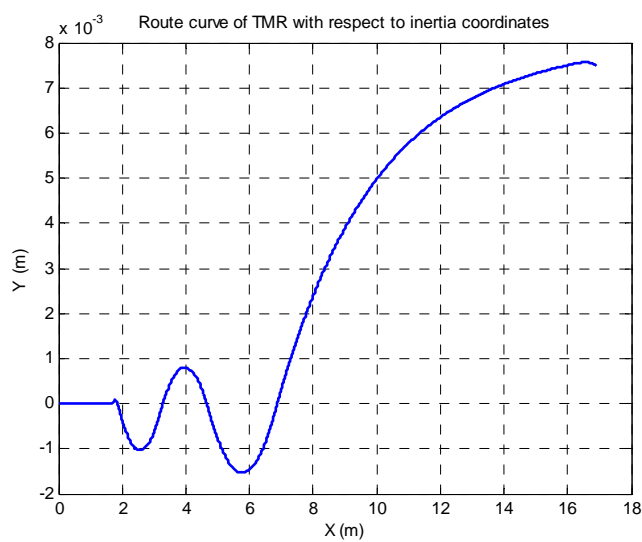

Fig.19. Results of path for $\mathrm{K}=20$

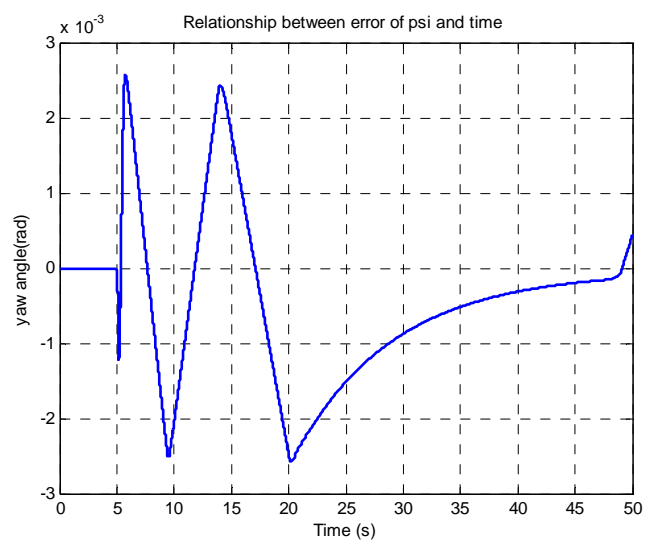

Fig.20. Results of error of $\psi$ for $\mathrm{K}=20$

\section{Conclusions}

In this paper, the mechanisms of two kinds of disturbances applied on UGV have been thoroughly discussed, which reveal the deviational rules of UGV. With the simulated case studies, disturbances of slope force and general disturbing on yaw motion represent characteristics individually.

As a test on first step, a PID controller (PD plus P control, in fact) is realized to keep the stability of motion for UGV. Simulation results prove the validity for disturbance incited by slope force, but also reveal the lack for general disturbance on yaw motion.

Finally, a hierarchical fuzzy controller combined with PID controller is proposed to compensate the lack of PID on disturbance on yaw motion. The PID controller in lower level is set to compensate the disturbance of slope force, and the differences between the model and the real UGV are feedback to the fuzzy logic controller on top level, which work out the adjustment that the UGV should take and guide the UGV maintain on the stable state. Simulation results demonstrate the power of the newly designed fuzzy controller.

\section{Acknowledgment}

Yue Ma thanks for the generous support from his colleagues on their wisdom suggestions.

\section{References}

1. G. A. Bekey, Autonomous Robots: From Biological Inspiration to Implementation and Control: MIT Press, 2005.

2. A. M. Lyapunov, Stability of motion: London, 1966.

3. N. V. Faldin, A. V. Morzhov, and I. M. Boiko, "Stability of Periodic Motions in Relay Feedback Systems with Saturation in Plant Dynamics," in American Control Conference, 2007. ACC '07, 2007, pp. 4721-4726.

4. T. Shibata and T. Murakami, "Null Space Motion Control by PID Control Considering Passivity in Redundant Manipulator," Industrial Informatics, IEEE Transactions on, vol. 4, pp. 261-270, 2008.

5. J. Zhao and Y. Yao, "On the Sudden Change in Joint Velocity during Fault Tolerant Operations for Spatial Coordinating Redundant Manipulators," in Intelligent Robots and Systems, 2006 IEEE/RSJ International Conference on, 2006, pp. 3885-3890.

6. Wuhong Wang, Huachun Tan, Heiner Bubb, "A framework for function allocation in intelligent driver interface design for comfort and safety," International Journal of Computational Intelligence Systems, vol. 3(5), pp. 531-541, 2010.

7. Wuhong Wang, Hongwei Guo,Heiner Bubb,Katsushi Ikeuchi, "A safety-based behavioural approaching model with various driving characteristics," Transportation Research Part C-Emerging Technologies, pp. 1248-1262, 2011.02.002.

8. Wuhong Wang, Jin Jing, Xiao Wang, Hongwei Guo, Xuemei Ren, Ikeuchi Katsushi, "Driver's various information process and multi-ruled decision-making mechanism: a fundamental of intelligent driving shaping model," International Journal of Computational Intelligence Systems, vol. 4(3), pp. 297-305, 2011.

9. K. Kin, O. Yano, and H. Urabe, "Enhancements in vehicle stability and steerability with slip control," JSAE Review, vol. 24, pp. 71-79, 2003

10. M. Shino and M. Nagai, "Independent wheel torque control of small-scale electric vehicle for handling and stability improvement," JSAE Review, vol. 24, pp. 449456, 2003.

11. Y. Ma, Zhu, Q.M., A., Winfield, Yan, Q. D., "Simulation research on braking performance of hydrodynamic torque converter and retarder based on automatic shifting rules," nt. J. Modelling, Identification and Control, vol. Vol. 8, pp. 80-87, 2009.

12. Z. Gong, J. I. Guzman, S. S. J., R. David C, G Dissanayake, and Hugh, "A Heuristic Rule-Based Switching and Adaptive PID Controller for a Large Autonomous Tracked Vehicle: From Development to Implementation," Proceedings of the 2004 IEEE International Conference on Control Applications Taipei, Taiwan, vol. 8633, pp. 1272-1277, 2004.

13. J. E. Normey-Rico, I. Alcalá, J. Gómez-Ortega, and E. F. Camacho, "Mobile robot path tracking using a robust PID 
controller," Control Engineering Practice, vol. 9, pp. 12091214, 2001.

14. H. R.W., R. A.L., and R. S.I., "Algorithms and sensors for small robot path following," in Proceedings ICRA 02, IEEE International Conference on Robotics and Automation, 2002, pp. 3850-3857.

15. T.-J. Rena, T.-C. Chen, and C.-J. Chen, "Motion control for a two-wheeled vehicle using a self-tuning PID controller," Control Engineering Practice, vol. 16, pp. 365375, 2008.

16. J. Ye, "Adaptive control of nonlinear PID-based analog neural networks for a nonholonomic mobile robot," Neurocomputing, vol. 71, pp. 1561-1565, 2008.

17. K. J. Astrom and R. M. Murray, Feedback Systems: An Introduction for Scientists and Engineers: Princeton University Press, April 1, 2008. 